\title{
Yang-Lee zeros of the one-dimensional $Q$-state Potts model
}

\author{
Seung-Yeon Kim* \\ School of Computational Sciences, Korea Institute for Advanced Study, \\ 207-43 Cheongryangri-dong, Dongdaemun-gu, Seoul 130-722, Korea
}

\begin{abstract}
The distributions of the Yang-Lee zeros of the ferromagnetic and antiferromagnetic $Q$-state Potts models in one dimension are studied for arbitrary $Q$ and temperature. The Yang-Lee zeros of the Potts antiferromagnet have been fully investigated for the first time. The distributions of the Yang-Lee zeros show a variety of different shapes. Some of the Yang-Lee zeros lie on the positive real axis even for $T>0$. For the ferromagnetic model this happens only for $Q<1$, while there exist some zeros of the antiferromagnetic model on the positive real axis both for $Q<1$ and for $Q>1$.

PACS numbers: 05.50.+q; 64.60.Cn; 75.10.Hk
\end{abstract}

Keywords: Potts model; Partition function zeros; Ferromagnetic Yang-Lee zeros; Antiferromagnetic YangLee zeros

\footnotetext{
* Electronic address: sykim@kias.re.kr
} 


\section{INTRODUCTION}

The $Q$-state Potts model [1, 2, 3, 4, 5] is a generalization of the Ising $(Q=2)$ model. The $Q$-state Potts model exhibits a rich variety of critical behavior and is very fertile ground for the analytical and numerical investigations of first- and second-order phase transitions. The Potts model is also related to other outstanding problems in physics and mathematics. Fortuin and Kasteleyn [6, 7] have shown that the $Q$-state Potts model in the limit $Q \rightarrow 1$ defines the problem of bond percolation. They [7] also showed that the problem of resistor network is related to a $Q=0$ limit of the partition function of the Potts model. In addition, the zero-state Potts model describes the statistics of treelike percolation [8], and is equivalent to the undirected Abelian sandpile model 9]. The $Q=\frac{1}{2}$ state Potts model has a connection to a dilute spin glass [10]. The $Q$-state Potts model with $0 \leq Q \leq 1$ describes transitions in the gelation and vulcanization processes of branched polymers [11]. The partition function of the Potts model is also known as the Tutte dichromatic polynomial [12] or the Whitney rank function [13] in graph theory and combinatorics of mathematics.

By introducing the concept of the zeros of the partition function in the complex magneticfield plane, Yang and Lee [14] proposed a mechanism for the occurrence of phase transitions in the thermodynamic limit and yielded a new insight into the unsolved problem of the ferromagnetic Ising model in an arbitrary nonzero external magnetic field. It has been known [14, 15, 16, 17, 18, 19, 20, 21, 22, 23, 24] that the distribution of the zeros of a model determines its critical behavior. Lee and Yang [15] also formulated the celebrated circle theorem which states that the partition function zeros of the Ising ferromagnet lie on the unit circle in the complex magnetic-field $\left(x=e^{\beta H}\right)$ plane. However, for the ferromagnetic $Q$-state Potts model with $Q>2$ the Yang-Lee zeros in the complex $x$ plane lie close to, but not on, the unit circle with the two exceptions of the critical point $x=1(H=0)$ itself and the zeros in the limit $T=0$ [19, 25, 26, 27, 28]. It has been shown [27, 28] that the distributions of the ferromagnetic Yang-Lee zeros for $Q>1$ have similar properties independent of dimension.

Recently, the exact results on the Yang-Lee zeros of the ferromagnetic Potts model have been found using the one-dimensional model. Mittag and Stephen [29] studied the Yang-Lee zeros of the three-state Potts ferromagnet in one dimension. Glumac and Uzelac [30] found the eigenvalues of the transfer matrix of the one-dimensional Potts model for general $Q$. 
In particular, they have shown that the ferromagnetic Yang-Lee zeros can lie on the real axis for $Q<1$. However, the properties of the Yang-Lee zeros of the antiferromagnetic Potts model have never been known except for the one-dimensional Ising antiferromagnet 31]. In this paper, we study the ferromagnetic and antiferromagnetic Yang-Lee zeros of the one-dimensional $Q$-state Potts model.

\section{PARTITION FUNCTION}

The $Q$-state Potts model in an external magnetic field $H_{q}$ on a lattice $G$ with $N_{s}$ sites and $N_{b}$ bonds is defined by the Hamiltonian

$$
\mathcal{H}_{Q}=-J \sum_{\langle i, j\rangle} \delta\left(\sigma_{i}, \sigma_{j}\right)-H_{q} \sum_{k} \delta\left(\sigma_{k}, q\right),
$$

where $J$ is the coupling constant (ferromagnetic model for $J>0$ and antiferromagnetic model for $J<0),\langle i, j\rangle$ indicates a sum over nearest-neighbor pairs, $\sigma_{i}=1,2, \ldots, Q$, and $q$ is a fixed integer between 1 and $Q$. The partition function of the model is

$$
Z_{Q}=\sum_{\left\{\sigma_{n}\right\}} e^{-\beta \mathcal{H}_{Q}},
$$

where $\left\{\sigma_{n}\right\}$ denotes a sum over $Q^{N_{s}}$ possible spin configurations and $\beta=\left(k_{B} T\right)^{-1}$. The partition function can be written as

$$
Z(a, x, Q)=\sum_{E=0}^{N_{b}} \sum_{M=0}^{N_{s}} \Omega_{Q}(E, M) a^{E} x^{M},
$$

where $a=y^{-1}=e^{\beta J}, x=e^{\beta H_{q}}, E$ and $M$ are positive integers $0 \leq E \leq N_{b}$ and $0 \leq M \leq N_{s}$, respectively, and $\Omega_{Q}(E, M)$ is the number of states with fixed $E$ and fixed $M$. The states with $E=0\left(E=N_{b}\right)$ correspond to the antiferromagnetic (ferromagnetic) ground states. For ferromagnetic interaction $J>0$ the physical interval is $1 \leq a \leq \infty(\infty \geq T \geq 0)$, whereas for antiferromagnetic interaction $J<0$ the physical interval $0 \leq a \leq 1(0 \leq T \leq \infty)$. The parameter $Q$ enters the Potts model as an integer. However, the study of the $Q$-state Potts model has been extended to continuous $Q$ due to the Fortuin-Kasteleyn representation of the partition function [6, 7] and its extension [32].

For the one-dimensional Potts model in an external field the eigenvalues of the transfer matrix were found by Glumac and Uzelac [30]. The eigenvalues are $\lambda_{ \pm}=(A \pm i B) / 2$, where 
$A=a(1+x)+Q-2$ and $B=-i \sqrt{[a(1-x)+Q-2]^{2}+4(Q-1) x}$, and $\lambda_{0}=a-1$ which is $(Q-2)$-fold degenerate. The partition function of the one-dimensional model $\left(N=N_{s}=N_{b}\right)$ is given by

$$
Z_{N}(a, x, Q)=\lambda_{+}^{N}+\lambda_{-}^{N}+(Q-2) \lambda_{0}^{N}
$$

\section{PARTITION FUNCTION ZEROS}

When $\lambda_{+}$and $\lambda_{-}$are two dominant eigenvalues, the partition function becomes

$$
Z_{N} \simeq \lambda_{+}^{N}+\lambda_{-}^{N}
$$

for large $N$. If we define $A=2 C \cos \psi$ and $B=2 C \sin \psi$, where $C=\sqrt{(a-1)(a+Q-1) x}$, then $\lambda_{ \pm}=C \exp ( \pm i \psi)$, and the partition function is

$$
Z_{N}=2 C^{N} \cos N \psi
$$

The zeros of the partition function are then given by

$$
\psi=\psi_{k}=\frac{2 k+1}{2 N} \pi, \quad k=0,1,2, \ldots, N-1
$$

In the thermodynamic limit the locus of the partition function zeros is determined by the solution of

$$
A=2 C \cos \psi
$$

where $0 \leq \psi \leq \pi$. In the special case $Q=2$ the contribution by the eigenvalue $\lambda_{0}$ disappears from the partition function, Eq. (4), and the equation (8) determines all the locus even for finite systems. From Eq. (8) the locus of the Yang-Lee zeros for any $Q$ is obtained to be

$$
x_{1}(\psi)=\frac{1}{a^{2}}\left[\sqrt{f_{1}} \cos \psi+i \sqrt{f_{2}}\right]^{2},
$$

where $f_{1}=(a-1)(a+Q-1)$ and $f_{2}=f_{1} \sin ^{2} \psi+Q-1$. The edge zeros of $x_{1}(\psi)$ are given by

$$
x_{ \pm}=\frac{1}{a^{2}}[\sqrt{(a-1)(a+Q-1)} \pm \sqrt{1-Q}]^{2}
$$

from $x_{1}(0)$ and $x_{1}(\pi)$. If $f_{1}>0$ and $f_{2}>0$ or $f_{1}<0$ and $f_{2}<0$, it is easily verified that

$$
\left|x_{1}(\psi)\right|=\sqrt{x_{+} x_{-}}=\left|\frac{a+Q-2}{a}\right| .
$$


From Eq. (11) we see that the zeros of $x_{1}(\psi)$ lie on a circle in the complex $x$ plane. The one point of the circle $x_{1}(\psi)$,

$$
x_{1}\left(\frac{\pi}{2}\right)=-\frac{a+Q-2}{a},
$$

always lies on the real axis. However, if $f_{1}>0$ and $f_{2}<0$ or $f_{1}<0$ and $f_{2}>0$, the zeros of $x_{1}(\psi)$ lie on the real axis. Recently, Ghulghazaryan et al. [33] tried to understand the properties of Eq. (9) in the antiferromganetic $(a \leq 1)$ region.

On the other hand, when $\lambda_{+}$and $\lambda_{0}$ are two dominant eigenvalues, the partition function can be written as

$$
Z_{N} \simeq \lambda_{+}^{N}+(Q-2) \lambda_{0}^{N}
$$

for large $N$. The partition function zeros are then determined by

$$
\frac{\lambda_{+}}{\lambda_{0}}=(2-Q)^{1 / N} \exp (i \phi),
$$

where

$$
\phi=\phi_{k}=\frac{2 \pi k}{N}, \quad k=0,1,2, \ldots, N-1
$$

In the thermodynamic limit the locus of the partition function zeros is determined by the solution of

$$
a^{2} x^{2}+(Q-1) x-a x A+(a-1) A e^{i \phi}-(a-1)^{2} e^{2 i \phi}=0,
$$

where $0 \leq \phi \leq 2 \pi$. The equation (16) also determines the locus of the zeros when $\lambda_{-}$and $\lambda_{0}$ are two dominant eigenvalues. Eq. (16) gives the second locus of the Yang-Lee zeros

$$
x_{2}(\phi)=\frac{e^{i \phi}\left[a+Q-2-(a-1) e^{i \phi}\right]}{a+Q-1-a e^{i \phi}},
$$

which is the unit circle in the limit $a \rightarrow \infty$ and a type of limaçon of Pascal for $a=0$. The two points of the locus $x_{2}(\phi)$,

$$
x_{2}(0)=1
$$

and

$$
x_{2}(\pi)=-\frac{2 a+Q-3}{2 a+Q-1},
$$

can be lie on the real axis. From $\left|\lambda_{ \pm}\right|=\left|\lambda_{0}\right|$ we also obtain

$$
x_{*}=\frac{a-1}{a+Q-1} .
$$

The second locus, Eq. (17), of the Yang-Lee zeros, although it is very important in understanding the $Q$-state Potts model, has never been considered in the literature until now. 


\section{FERROMAGNETIC $(a \geq 1)$ YANG-LEE ZEROS}

In this section we study the Yang-Lee zeros of the ferromagnetic $Q$-state Potts model for $a \geq 1$.

\section{A. $Q>1$}

In this case $f_{1}$ and $f_{2}$ are always positive and the Yang-Lee zeros $x_{1}(\psi)$ lie on a circle where the eigenvalues satisfy

$$
\frac{\left|\lambda_{ \pm}\right|}{\left|\lambda_{0}\right|}=\sqrt{\frac{(a+Q-1)(a+Q-2)}{a(a-1)}}>1,
$$

which implies that the locus $x_{2}(\phi)$ does not appear. For $1<Q<2$ the zeros lie inside the unit circle while for $Q>2$ the zeros lie outside the unit circle, as shown in 27, 30]. In the special case $Q=2$ we of course find that $\left|x_{1}(\psi)\right|=1$, as proved by Lee and Yang [15].

The argument $\theta$ of $x_{1}(\psi)\left(=\left|x_{1}(\psi)\right| e^{i \theta(\psi)}\right)$ is given by

$$
\cos \frac{\theta}{2}=\sqrt{\frac{(a-1)(a+Q-1)}{a(a+Q-2)}} \cos \psi .
$$

As $a \rightarrow \infty(T \rightarrow 0)$, the zeros approach the unit circle. For $a=1(T=\infty), \cos (\theta / 2)=0$, so $\theta(\psi)=\pi$ and all the zeros lie at $1-Q$. As $Q \rightarrow \infty$, the radius $\left|x_{1}(\psi)\right|$ increases without bound. The Yang-Lee edge zero for $T>0$ is given by

$$
\theta_{0}=2 \cos ^{-1} \sqrt{\frac{(a-1)(a+Q-1)}{a(a+Q-2)}}>0,
$$

while $\theta_{0}=0$ at $T=0$. Therefore, we conclude that no zero lie on the positive real axis for any $T>0$.

\section{B. $Q<1$}

At $a=1(T=\infty)$, all the Yang-Lee zeros lie at the point $1-Q$ on the positive real axis. For $1<a \leq 1-\frac{Q}{2}+\frac{\sqrt{Q}}{2}$, all the zeros lie on the positive real axis between $x_{-}$and $x_{+}$

$\left(0<x_{-}<x_{+}<1\right)$, as pointed out by Glumac and Uzelac [30, 33, 34]. For $a>1-\frac{Q}{2}+\frac{\sqrt{Q}}{2}$, the locus consists of the loop $x_{2}(\phi)\left(\phi_{*} \leq \phi \leq 2 \pi-\phi_{*}\right)$ and the line $x_{1}(\psi)\left(0 \leq \psi \leq \psi_{*}\right)$ on 
the positive real axis between $x_{*}=x_{1}\left(\psi_{*}\right)$ and $x_{+}=x_{1}(0)\left(0<x_{*}<x_{+}<1\right)$. The loop $x_{2}$ meets with the line $x_{1}$ at the point

$$
x_{*}=x_{2}\left(\phi_{*}\right)=x_{2}\left(2 \pi-\phi_{*}\right)
$$

where $\left|\lambda_{+}\right|=\left|\lambda_{-}\right|=\left|\lambda_{0}\right|$. The loop $x_{2}(\phi)$ cuts the real axis at two points $x_{2}(\pi)$ and $x_{*}$ $\left(>x_{2}(\pi)\right)$. The sign of $x_{2}(\pi)$ is positive for $1-\frac{Q}{2}+\frac{\sqrt{Q}}{2}<a<\frac{3-Q}{2}$ and negative for $a>\frac{3-Q}{2}$. Figure 1 shows the locus of the Yang-Lee zeros for $Q=\frac{1}{2}$ and $a=\frac{6}{5}$ from which we obtain $x_{2}(\pi)=\frac{1}{19}, x_{*}=\frac{2}{7}$, and $x_{+}=0.8119$.

At $Q=0$, the line $x_{1}(\psi)$ shrink to the point

$$
x_{*}=x_{+}=x_{2}(0)=1 \text {, }
$$

and all the zeros lie on the loop $x_{2}(\phi)(0 \leq \phi \leq 2 \pi)$ for $a>1$. As $Q \rightarrow 1$, two points $x_{*}$ and $x_{+}$approach $(a-1) / a$ together, and the line $x_{1}(\psi)$ disappears. At $a=\infty(T=0)$, the line $x_{1}(\psi)$ again shrink to the point $x_{*}=x_{+}=1$, and the loop $x_{2}(\phi)$ becomes the unit circle centered at the origin.

\section{ANTIFERROMAGNETIC $(0 \leq a \leq 1)$ YANG-LEE ZEROS}

In this section we investigate the Yang-Lee zeros of the antiferromagnetic $Q$-state Potts model for $0 \leq a \leq 1$.

\section{A. $Q>1$}

Because $f_{1}$ is always negative, the zeros of $x_{1}(\psi)$ lie on a circle if $f_{2}<0\left(\psi_{0}<\psi<\pi-\psi_{0}\right)$ and on the real axis if $f_{2}>0\left(0 \leq \psi<\psi_{0}\right.$ or $\left.\pi-\psi_{0}<\psi \leq \pi\right)$. For $a<1-\frac{Q}{2}$, the locus consists of the line $x_{1}(\psi)\left(0 \leq \psi \leq \psi_{0}\right.$ and $\left.\pi-\psi_{0} \leq \psi \leq \psi_{*}\right)$ on the negative real axis between $x_{+}=x_{1}(0)$ and $x_{*}=x_{1}\left(\psi_{*}\right)\left(x_{+}<x_{*}<0\right)$, the circle $x_{1}(\psi)\left(\psi_{0} \leq \psi \leq \pi-\psi_{0}\right)$ with the radius $\left|x_{1}(\psi)\right|$, and the loop $x_{2}(\phi)\left(0 \leq \phi \leq \phi_{*}\right.$ and $\left.2 \pi-\phi_{*} \leq \phi \leq 2 \pi\right)$, inside the circle $x_{1}$. The loop $x_{2}$ again meets with the line $x_{1}$ at the point

$$
x_{*}=x_{2}\left(\phi_{*}\right)=x_{2}\left(2 \pi-\phi_{*}\right)
$$


where $\left|\lambda_{+}\right|=\left|\lambda_{-}\right|=\left|\lambda_{0}\right|$. The circle cuts the real axis at two points $x_{1}^{0}$ and $x_{1}\left(\frac{\pi}{2}\right)\left(=-x_{1}^{0}>\right.$ 1 ), where the point $x_{1}^{0}$ is defined by

$$
x_{1}^{0}=x_{1}\left(\psi_{0}\right)=x_{1}\left(\pi-\psi_{0}\right)=\frac{a+Q-2}{a} .
$$

Similarly, the loop also cuts the real axis at two points $x_{*}(<0)$ and $x_{2}(0)(=1)$. At $a=0$ $(T=0)$, the circle $x_{1}(\psi)$ disappears for $1<Q<2$, and the locus consists of the line $x_{1}(\psi)$ on the real axis between $-\infty$ and $x_{*}(<0)$ and the loop $x_{2}(\phi)$. For example, figure 2 shows the locus for $Q=\frac{11}{10}$ and $a=\frac{1}{10}$. In this case we obtain $x_{+}=-54.83, x_{1}^{0}=-8, x_{*}=-\frac{9}{2}$, the radius $\left|x_{1}\right|=8$ for the circle, $\psi_{0}=48.19^{\circ}, \psi_{*}=134.07^{\circ}$, and $\phi_{*}=46.02^{\circ}$.

At $a=1-\frac{Q}{2}$, two points $x_{*}$ and $x_{1}^{0}$ on the real axis meet at

$$
x_{*}=x_{1}^{0}=-1
$$

other two points $x_{1}\left(\frac{\pi}{2}\right)$ and $x_{2}(0)$ on the real axis also meet at

$$
x_{1}\left(\frac{\pi}{2}\right)=x_{2}(0)=1
$$

and the circle $x_{1}(\psi)$ and the loop $x_{2}(\phi)$ become the identical locus as the unit circle. On this unit circle, three eigenvalues have the same magnitude

$$
\left|\lambda_{+}\right|=\left|\lambda_{-}\right|=\left|\lambda_{0}\right|=\frac{Q}{2}
$$

Therefore, the locus consists of the line $x_{1}(\psi)$ on the real axis between $x_{+}$and $x_{*}$ and the circle.

In the region $1-\frac{Q}{2}<a<1-\frac{Q}{2}+\frac{\sqrt{Q}}{2}$, the circle $x_{1}(\psi)$ disappears, and the line $x_{1}(\psi)$ $\left(0 \leq \psi \leq \psi_{*}\right)$ on the negative real axis between $x_{+}$and $x_{*}\left(x_{+}<x_{*}<0\right)$ again meets with the loop $x_{2}(\phi)\left(\phi_{*} \leq \phi \leq 2 \pi-\phi_{*}\right)$ at the point $x_{*}$. The loop cuts the real axis at two points $x_{*}$ and $x_{2}(\pi)$. The sign of $x_{2}(\pi)$ is positive $\left(0<x_{2}(\pi)<1\right)$ for $1-\frac{Q}{2}<a<\frac{3-Q}{2}$ and negative for $\frac{3-Q}{2}<a<1-\frac{Q}{2}+\frac{\sqrt{Q}}{2}$. Figure 3 shows the locus of the Yang-Lee zeros for $Q=3$ and $a=\frac{1}{10}$ which give $x_{2}(\pi)=-\frac{1}{11}, x_{*}=-\frac{3}{7}$, and $x_{+}=-777.84$. At $a=0(T=0)$, the locus still consists of the line $x_{1}(\psi)$ on the real axis between $-\infty$ and $x_{*}(<0)$ and the loop $x_{2}(\phi)$ for $2<Q<4$. At $T=0$, the sign of $x_{2}(\pi)$ is positive $\left(0 \leq x_{2}(\pi)<1\right)$ for $2<Q \leq 3$ and negative for $3<Q<4$. As $Q \rightarrow 1$, two points $x_{+}$and $x_{*}$ approach $(a-1) / a$ together, and the line $x_{1}(\psi)$ disappears.

At $a=1-\frac{Q}{2}+\frac{\sqrt{Q}}{2}, x_{*}=x_{-}$, and the loop $x_{2}(\phi)$ shrinks to the point $x_{*}$. For $a>1-\frac{Q}{2}+\frac{\sqrt{Q}}{2}$, the loop disappears, and the only locus is the line $x_{1}(\psi)$ on the negative real axis between 
$x_{+}$and $x_{-}\left(x_{+}<x_{-}<0\right)$. As $Q \rightarrow \infty, x_{ \pm} \rightarrow-\infty$ for $a<1$. At $a=1(T=\infty)$, two edge zeros $x_{+}$and $x_{-}$meet, and the line $x_{1}(\psi)$ shrinks to the point

$$
x_{+}=x_{-}=1-Q
$$

for $Q>1$. In the special case $Q=2, f_{1}<0$ and $f_{2}>0$, and the line $x_{1}(\psi)$ is the only locus of the Yang-Lee zeros. Therefore, all the Yang-Lee zeros of the antiferromagnetic Ising model lie on the negative real axis between $x_{+}$and $x_{-}\left(>x_{+}\right)$for $a<1$, as shown by Yang 31]. At $a=0(T=0)$, all the zeros lie on the negative real axis between two edge zeros,

$$
x_{a}=-\infty
$$

and

$$
x_{b}=\frac{(Q-2)^{2}}{4(1-Q)} \quad\left(x_{b} \leq 0\right)
$$

for $Q=2$ and $Q \geq 4$.

\section{B. $Q<1$}

For $0 \leq a<1-\frac{Q}{2}-\frac{\sqrt{Q}}{2}$, the locus consists of the loop $x_{2}(\phi)\left(0 \leq \phi \leq \phi_{*}\right.$ and $2 \pi-\phi_{*} \leq$ $\phi \leq 2 \pi)$ and the line $x_{1}(\psi)\left(0 \leq \psi \leq \psi_{*}\right)$ on the positive real axis between $x_{*}=x_{1}\left(\psi_{*}\right)$ and $x_{+}=x_{1}(0)\left(1<x_{*}<x_{+}\right)$. The loop $x_{2}$ cuts the real axis at two points $x_{2}(0)(=1)$ and $x_{*}$ $(>1)$. The loop again meets with the line $x_{1}$ at the point

$$
x_{*}=x_{2}\left(\phi_{*}\right)=x_{2}\left(2 \pi-\phi_{*}\right),
$$

where $\left|\lambda_{+}\right|=\left|\lambda_{-}\right|=\left|\lambda_{0}\right|$. At $Q=0$, two points $x_{2}(0)$ and $x_{*}$ meet, the loop $x_{2}(\phi)$ shrinks to the point $x_{2}(0)=x_{*}$, and all the Yang-Lee zeros lie on the positive real axis between $x_{-}$ $\left(=x_{*}=1\right)$ and $x_{+}\left(\geq x_{-}\right)$for $a \leq 1$.

At $a=1-\frac{Q}{2}-\frac{\sqrt{Q}}{2}, x_{*}=x_{+}$, and the line $x_{1}(\psi)$ shrinks to the point $x_{*}$. In the region $1-\frac{Q}{2}-\frac{\sqrt{Q}}{2}<a<1-\frac{Q}{2}$, the line disappears, and the locations of Yang-Lee zeros are completely determined by the locus $x_{2}(\phi)(0 \leq \phi \leq 2 \pi)$ which cuts the real axis at two points $x_{2}(0)(=1)$ and $x_{2}(\pi)(>1)$. The shape of the locus $x_{2}(\phi)$ is changed from a waterdrop-like shape for $1-\frac{Q}{2}-\frac{\sqrt{Q}}{2}<a<1-Q$ through a circle with center $\frac{1}{1-Q}$ and radius $\frac{Q}{1-Q}$ for $a=1-Q$ to a crescent-like shape for $1-Q<a<1-\frac{Q}{2}$. Figure 4 shows the locus of the Yang-Lee zeros for $Q=\frac{9}{10}$ and $a=\frac{2}{5}$ which give $x_{2}(\pi)=\frac{13}{7}$. 
At $a=1-\frac{Q}{2}$, the loci $x_{1}(\psi)$ and $x_{2}(\phi)$ become identical to be the unit circle on which three eigenvalues again have the same magnitude

$$
\left|\lambda_{+}\right|=\left|\lambda_{-}\right|=\left|\lambda_{0}\right|=\frac{Q}{2}
$$

For $a>1-\frac{Q}{2}$, all the Yang-Lee zeros lie on the circle $x_{1}(\psi)(0 \leq \psi \leq \pi)$ which cuts the positive real axis at the point $x_{1}\left(\frac{\pi}{2}\right)\left(0<x_{1}\left(\frac{\pi}{2}\right)<1\right)$. At $a=1(T=\infty)$, this circle shrinks to the point $x_{1}(\psi)=1-Q$ for $Q<1$.

\section{ONE-STATE $(\mathrm{Q}=1)$ POTTS MODEL}

At $Q=1$, the partition function becomes

$$
Z_{N}(a, x, Q=1)=(a x)^{N}
$$

Therefore, all the Yang-Lee zeros lie on the point $x=0$. On the other hand, in the limit $Q \rightarrow 1$, the line $x_{1}(\psi)$ disappears, and the circle $x_{1}(\psi)$ is the uniform distribution of the zeros because the circle approaches

$$
x_{1}(\psi)=\left|\frac{a-1}{a}\right| e^{2 i \psi}
$$

The loop $x_{2}(\phi)$ also approaches

$$
x_{2}(\phi)=\frac{a-1}{a} e^{i \phi}
$$

which is the same as the circle $x_{1}(\psi)$.

\section{CONCLUSION}

We have studied the interesting properties of the ferromagnetic and antiferromagnetic Yang-Lee zeros of the one-dimensional $Q$-state Potts model for arbitrary $Q$ and temperature. It has been shown that the distributions of the Yang-Lee zeros have a variety of different shapes. In particular, the antiferromagnetic Yang-Lee zeros of the Potts model have been fully investigated for the first time. One of the most interesting results is that some of the Yang-Lee zeros lie on the positive real axis even for $T>0$. For the ferromagnetic Yang-Lee zeros this happens only for $Q<1$, while there exist some Yang-Lee zeros of the antiferromagnetic Potts model on the positive real axis both for $Q<1$ and for $Q>1$. 
The results obtained from the one-dimensional Potts model may be considered as a road to the full understanding of the Yang-Lee zeros in higher dimensions in that for $Q>1$ the distributions of the ferromagnetic Yang-Lee zeros have similar properties independent of dimension [27, 28].

\section{Acknowledgments}

The author is grateful to Prof. R.J. Creswick for very useful discussions. 
[1] R.B. Potts, Proc. Cambridge Philos. Soc. 48 (1952) 106.

[2] F.Y. Wu, Rev. Mod. Phys. 54 (1982) 235.

[3] F.Y. Wu, J. Appl. Phys. 55 (1984) 2421.

[4] R.J. Baxter, Exactly Solved Models in Statistical Mechanics, Academic Press, London, 1982.

[5] P.P. Martin, Potts Models and Related Problems in Statistical Mechanics, World Scientific, Singapore, 1991.

[6] P.W. Kasteleyn, C.M. Fortuin, J. Phys. Soc. Jpn. Suppl. 26 (1969) 11.

[7] C.M. Fortuin, P.W. Kasteleyn, Physica 57 (1972) 536.

[8] M.J. Stephen, Phys. Lett. A 56 (1976) 149.

[9] S.N. Majumdar, D. Dhar, Physica A 185 (1992) 129.

[10] A. Aharony, J. Phys. C 11 (1978) L457.

[11] T.C. Lubensky, J. Isaacson, Phys. Rev. Lett. 41 (1978) 829.

[12] W.T. Tutte, Proc. Cambridge Philos. Soc. 43 (1947) 26.

[13] H. Whitney, Ann. Math. 33 (1932) 688.

[14] C.N. Yang, T.D. Lee, Phys. Rev. 87 (1952) 404.

[15] T.D. Lee, C.N. Yang, Phys. Rev. 87 (1952) 410.

[16] C. Itzykson, R.B. Pearson, J.B. Zuber, Nucl. Phys. B 220 (1983) 415.

[17] R.J. Creswick, S.-Y. Kim, Phys. Rev. E 56 (1997) 2418.

[18] R.J. Creswick, S.-Y. Kim, in: D.P. Landau, K.K. Mon, H.-B. Schüttler (Eds.), Computer Simulation Studies in Condensed-Matter Physics, Vol. 10, Springer, Berlin, 1998, p. 224.

[19] R.J. Creswick, S.-Y. Kim, Comput. Phys. Commun. 121 (1999) 26.

[20] W. Janke, R. Kenna, J. Stat. Phys. 102 (2001) 1211.

[21] W. Janke, R. Kenna, Comput. Phys. Commun. 147 (2002) 443.

[22] W. Janke, R. Kenna, Nucl. Phys. B (Proc. Suppl.) 106 (2002) 905.

[23] N.A. Alves, J.P.N. Ferrite, U.H.E. Hansmann, Phys. Rev. E 65 (2002) 036110.

[24] R.A. Kromhout, Physica B 301 (2001) 389.

[25] S.-Y. Kim, R.J. Creswick, Phys. Rev. Lett. 81 (1998) 2000.

[26] S.-Y. Kim, R.J. Creswick, Phys. Rev. Lett. 82 (1999) 3924.

[27] S.-Y. Kim, R.J. Creswick, Physica A 281 (2000) 252. 
[28] S.-Y. Kim, Nucl. Phys. B 637 (2002) 409.

[29] L. Mittag, M.J. Stephen, J. Stat. Phys. 35 (1984) 303.

[30] Z. Glumac, K. Uzelac, J. Phys. A 27 (1994) 7709.

[31] C.N. Yang, Special Problems of Statistical Mechanics (Lectures at the University of Washington), 1952.

[32] H.W.J. Blöte, M.P. Nightingale, Physica A 112 (1982) 405.

[33] R.G. Ghulghazaryan, N.S. Ananikan, P.M.A. Sloot, Phys. Rev. E 66 (2002) 046110.

[34] J.L. Monroe, J. Phys. A 29 (1996) 5421. 


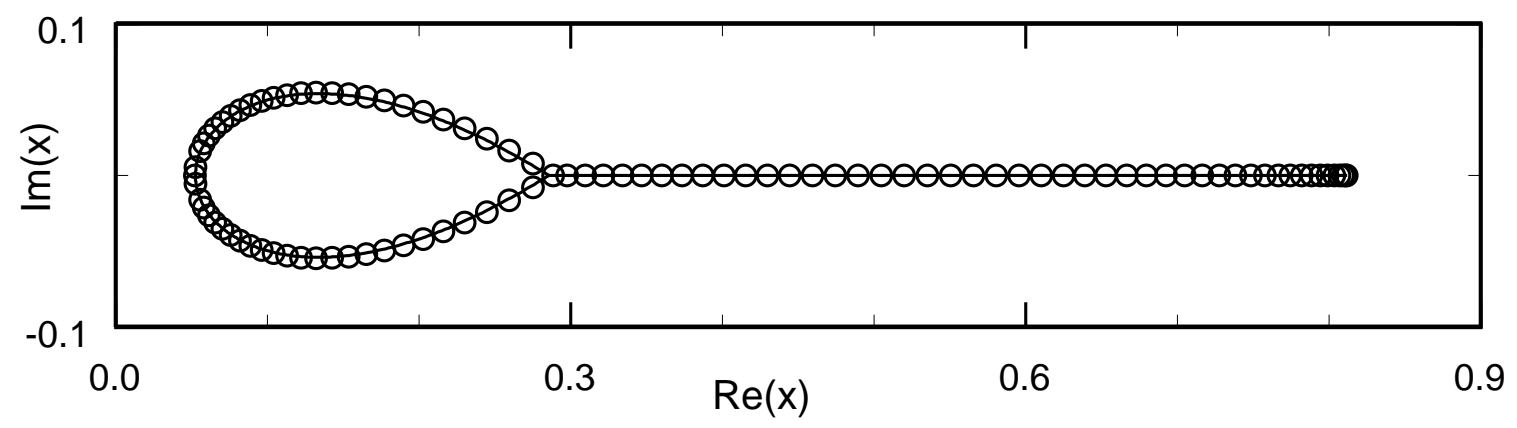

FIG. 1: The locus of the Yang-Lee zeros in the complex $x=e^{\beta H_{q}}$ plane for $Q=\frac{1}{2}$ and $a=e^{\beta J}=\frac{6}{5}$. For comparison, the zeros for a finite-size system $(N=100)$ are also shown (open circles).

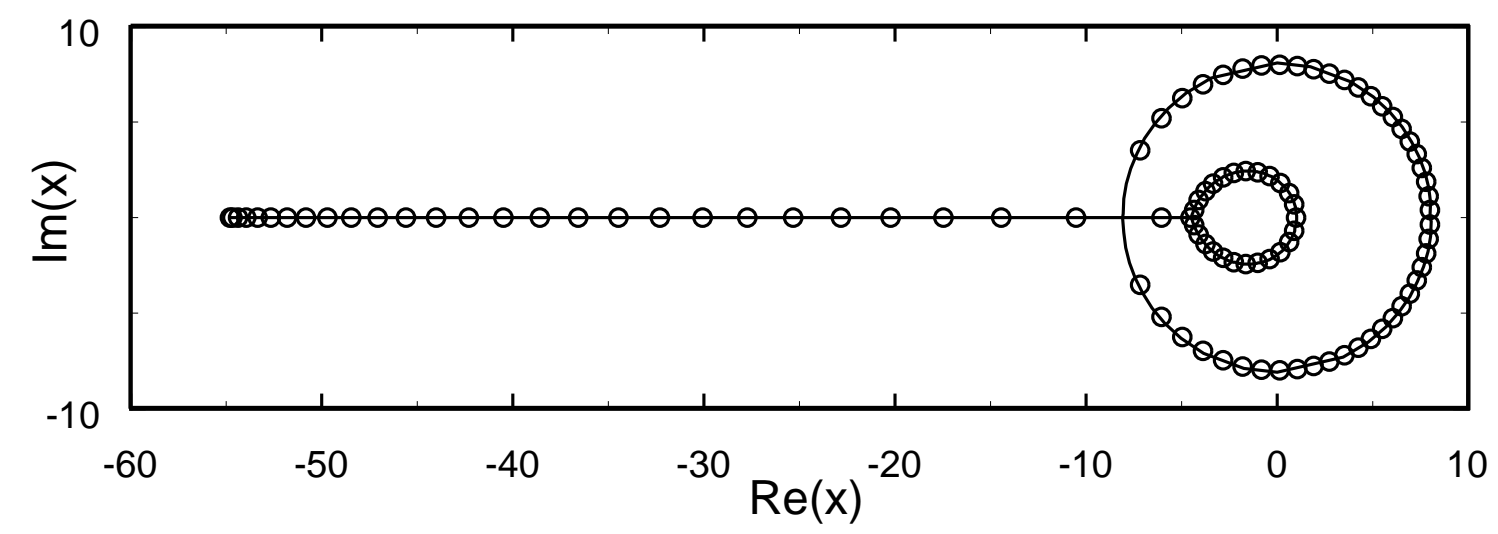

FIG. 2: The locus of the Yang-Lee zeros for $Q=\frac{11}{10}$ and $a=\frac{1}{10}$. 


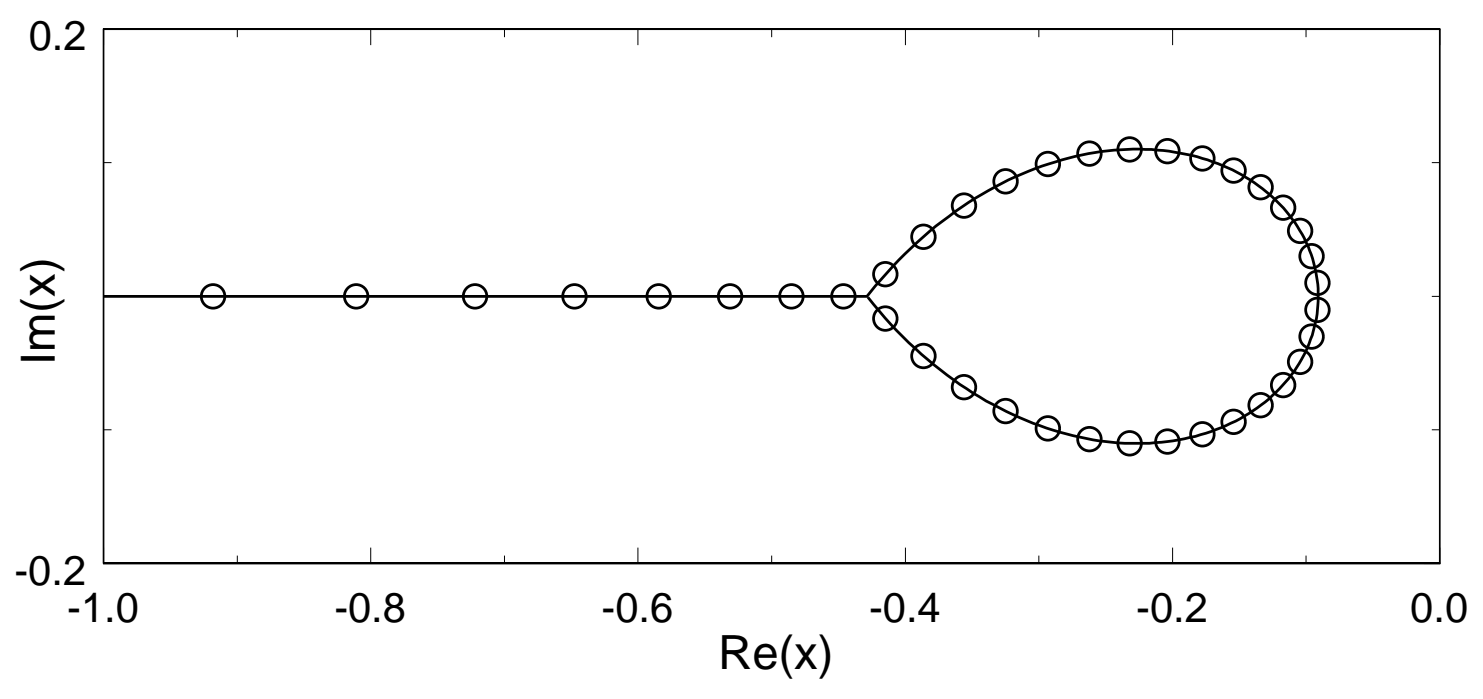

FIG. 3: The locus of the Yang-Lee zeros for $Q=3$ and $a=\frac{1}{10}$. The zeros on the real axis lie between $x_{*}=-\frac{3}{7}$ and $x_{+}=-777.84$. Most of them are omitted in the figure.

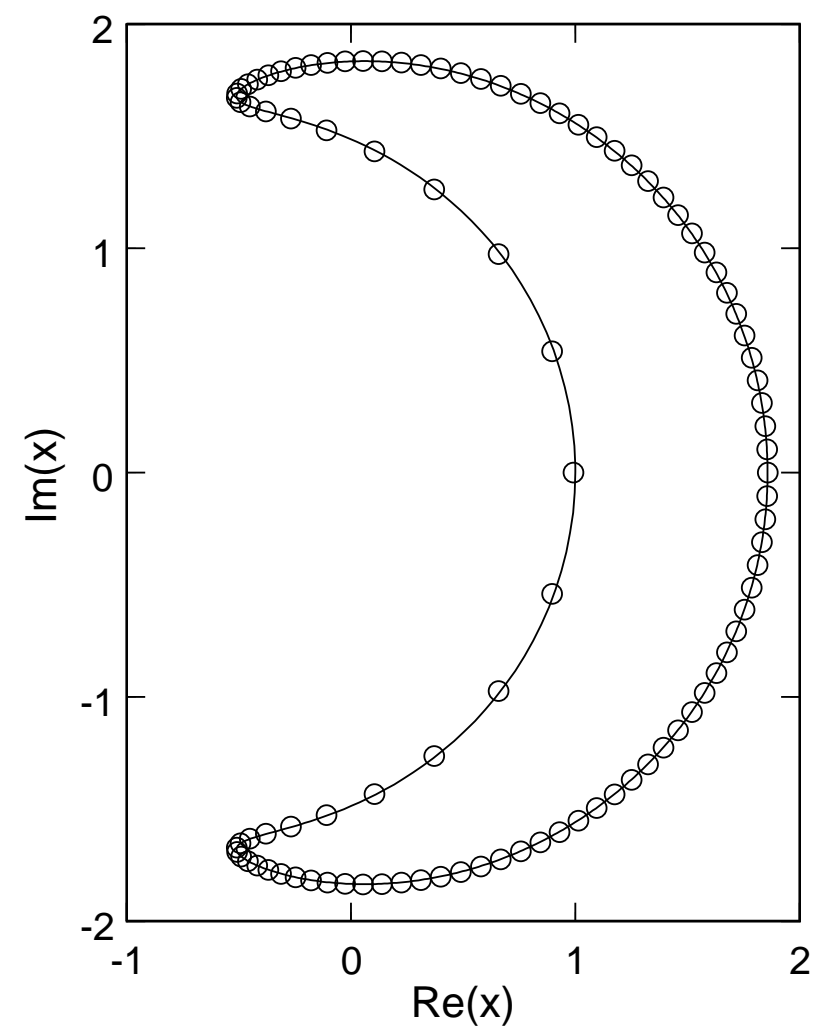

FIG. 4: The locus of the Yang-Lee zeros for $Q=\frac{9}{10}$ and $a=\frac{2}{5}$. 